\title{
THE
}

$11-1-1984$

\section{Susceptibilities of One-Dimensional Quantum Spin Models at Zero Temperature}

Gerhard Müller

University of Rhode Island, gmuller@uri.edu

Robert E. Shrock

Follow this and additional works at: https://digitalcommons.uri.edu/phys_facpubs

Terms of Use

All rights reserved under copyright.

\section{Citation/Publisher Attribution}

G. Müller and R.E. Shrock. Susceptibilities of one-dimensional quantum spin models at zero temperature. Phys. Rev. B 30 (1984), 5254-5264. doi: 10.1103/PhysRevB.30.5254

Available at http://dx.doi.org/10.1103/PhysRevB.30.5254.

This Article is brought to you for free and open access by the Physics at DigitalCommons@URI. It has been accepted for inclusion in Physics Faculty Publications by an authorized administrator of DigitalCommons@URI. For more information, please contact digitalcommons-group@uri.edu. 


\title{
Susceptibilities of one-dimensional quantum spin models at zero temperature
}

\author{
Gerhard Müller* and Robert E. Shrock \\ Institute for Theoretical Physics, State University of New York at Stony Brook, Stony Brook, New York 11794
}

(Received 9 May 1984)

\begin{abstract}
We calculate precise numerical values for the nondivergent direct or staggered zero-temperature susceptibilities of the one-dimensional, $S=\frac{1}{2}$, transverse Ising model at the critical field and for the isotropic $X Y$ model in zero field which have not been previously determined analytically. Our method is based on a rigorous approach to calculate dynamic correlation functions for these models. We also investigate the exact nature of the divergencies in the $q$-dependent susceptibilities. Our results are compared with existing predictions of approximate analytic approaches and numerical finite-chain calculations. Our result for the $X Y$ case is directly relevant for the interpretation of recent susceptibility measurements on the quasi-one-dimensional magnetic compound $\mathrm{Cs}_{2} \mathrm{CoCl}_{4}$.
\end{abstract}

\section{INTRODUCTION}

The theoretical study of one-dimensional (1D) quantum spin models continues to receive a great deal of impetus from three major sources: (i) the rewarding experience that there exist realistic but nontrivial many-body systems which are amenable to exact and rigorous analysis, (ii) the discovery that many of these models are related by virtue of various mappings to a rich variety of physical phenomena in addition to the quasi-1D magnetic systems which they describe directly; (iii) the ever more successful efforts by coordinate chemists and physicists to synthesize highquality quasi-1D magnetic compounds and the growing interest by solid-state experimentalists in their magnetic properties.

In this paper we are concerned with susceptibilities for 1D quantum spin models. Susceptibility measurements belong to the basic experimental techniques which are used to determine the detailed magnetic interaction in quasi-1D compounds. ${ }^{1}$ It is therefore crucial to have accurate results available for the susceptibilities of those 1D model systems which are supposed to be realized by actual physical compounds.

The static, wave-number-dependent susceptibility is defined by the linear response of a spin system to a static but, in general, inhomogeneous magnetic field:

$$
\chi_{\xi \xi^{\prime}}(q)=\partial M_{\xi}(q) / \partial h_{\xi^{\prime}}(q), \xi, \xi^{\prime}=x, y, z,
$$

where

$$
\begin{aligned}
& M_{\xi}(q) \equiv N^{-1} \sum_{l=1}^{N} e^{-i q l}\left\langle S_{l}^{\xi}\right\rangle, \\
& h_{\xi}(q)=N^{-1} \sum_{l=1}^{N} e^{-i q l} h_{l}^{\xi} .
\end{aligned}
$$

Here and in the following, the wave number $q$ denotes the projection of the 3D wave vector $\vec{Q}$ onto the direction $\hat{n}_{c}$ of the spin chain in the lattice: $q=\overrightarrow{\mathrm{Q}} \cdot \hat{n}_{c}$. In the following, the direction $\hat{n}_{c}$ is taken to be arbitrary with respect to the spin coordinates $\xi$. We shall concentrate on the diagonal response $\left(\xi=\xi^{\prime}\right)$ and on the specific wave numbers $q=0$ and $q=\pi$ corresponding to the direct susceptibility $\chi_{\xi \xi}$ and the staggered susceptibility $\bar{\chi}_{\xi \xi^{\prime}}$, respectively.

In this paper, we shall determine the staggered zerotemperature susceptibilities $\bar{\chi}_{x x}$ of the $1 \mathrm{D}, S=\frac{1}{2}$ transverse Ising (TI) model at the critical external magnetic field and the 1D, $S=\frac{1}{2}$, isotropic $X Y$ model in zero field, specified, respectively, by the Hamiltonians

$$
H_{\mathrm{TI}}=-J \sum_{l=1}^{N}\left(2 S_{l}^{x} S_{l+1}^{x}+h_{c} S_{l}^{z}\right), \quad h_{c}=1,
$$

and

$$
H_{X Y}=-J \sum_{l=1}^{N}\left(S_{l}^{x} S_{l+1}^{x}+S Y S_{l+1}^{y}\right)
$$

in the limit $N \rightarrow \infty$, with $J>0$ and periodic boundary conditions imposed. Both Hamiltonians (1.2) and (1.3) are special cases of the more general anisotropic $X Y(A-X Y)$ model in a magnetic field

$$
H_{A-X Y}=-J \sum_{l=1}^{N}\left[(1+\gamma) S_{l}^{x} S_{l+1}^{x}+(1-\gamma) S Y S_{l+1}^{y}+h S_{l}^{z}\right] \text {, }
$$

The $X Y$ Hamiltonian (1.3) is also noteworthy as the special case $\Delta=0$ of the $X X Z$ model

$$
H_{X X Z}=-J \sum_{l=1}^{N}\left(S_{l}^{x} S_{l+1}^{x}+S y S_{++1}^{y}+\Delta S_{l}^{z} S_{l+1}^{z}\right) .
$$

Some earlier studies of the models (1.2)-(1.5), in particular, their zero-temperature properties, include Refs. 2-28. Our new results represent important additions to the existing zero-temperature susceptibility results as obtained by previous exact and approximate analytic approaches and by numerical calculations, the latter mostly in the form of finite-chain calculations. ${ }^{3-5,8-10,12,14-16,20,23}$ Furthermore, our result for the $X Y$ case is directly relevant for the interpretation of recent susceptibility measurements ${ }^{23}$ on the quasi-1D compound $\mathrm{Cs}_{2} \mathrm{CoCl}_{4}$.

In Sec. II we present our new calculations and results for the staggered susceptibilities $\left(\bar{\chi}_{x x}\right)_{\mathrm{TI}}$ and $\left(\bar{\chi}_{x x}\right)_{X Y}$ of 
the two models (1.2) and (1.3), respectively. Section III contains a discussion of new results on the divergences in the $q$-dependent susceptibilities $\left[\chi_{x x}(q)\right]_{\mathrm{TI}}$ and $\left[\chi_{x x}(q)\right]_{X Y}$ and their implications for the form of the dynamic structure factors. In Sec. IV, finally, our results are compared with existing predictions based on finite-chain extrapolations and discussed in the context of other exactly known zero-temperature results for these models.

\section{THE STAGGERED SUSCEPTIBILITIES $\left(\bar{\chi}_{x x}\right)_{T I}$ and $\left(\bar{\chi}_{x x}\right)_{X Y}$ AT $T=0$}

\section{A. The method}

We begin this section by recalling that the staggered susceptibilities $\bar{\chi}_{x x}$ and $\bar{\chi}_{y y}$ of the TI model (1.2) and of the $X Y$ model (1.3), both with $J>0$, are equal to the direct susceptibilities $\chi_{x x}, \chi_{y y}$ of the same models with $J<0$, and vice versa. In contrast, the susceptibilities $\chi_{z z}$ and $\bar{\chi}_{z z}$ do not depend on the sign of $J$ for these models. ${ }^{12,13}$ Furthermore, we have $\chi_{x x}(q)=\chi_{y y}(q)$ $=\chi_{\perp \perp}(q)$ in the $X Y$ model for obvious symmetry reasons. For $J>0$ the direct susceptibility $\chi_{x x}$ diverges as $T \rightarrow 0$, whereas $\bar{\chi}_{x x}$ stays finite. At $T=0$ the $q$-dependent susceptibility $\chi_{x x}(q)$ has a power-law divergence for $q \rightarrow 0$. (See the discussion in Sec. IV for further details.) In order to determine the susceptibilities $\chi_{x x}$ and $\bar{\chi}_{x x}$ directly from the definition (1.1), it is necessary to solve the corresponding models in the presence of a magnetic field $h_{x}$. At present, however, such solutions do not exist for the TI or the $X Y$ model, except for very special circumstances ${ }^{21}$ which are not relevant here. The alternative, which in our case is feasible, is to determine the susceptibility from known correlation functions for these models at $h_{x}=0$ by invoking the fluctuation-dissipation theorem. Note, however, that in contrast to classical spin models at $T>0$, where the susceptibility can be inferred from static (equal-time) two-spin correlation functions, the same information for quantum spin systems can be derived, in general, only from time-dependent correlation functions. ${ }^{29}$ For the TI and the $X Y$ model we can take advantage of the fact that recent work has achieved a great deal of progress in the understanding of the time-dependent correlation functions

$$
\Xi_{n}(t)=4\left[\left\langle S_{\delta}^{\xi}(t) S_{n}^{\xi}\right\rangle-\left\langle S_{\gamma}^{\xi}\right\rangle\left\langle S_{n}^{\xi}\right\rangle\right], \quad \xi=x, y
$$

at zero temperature. ${ }^{24-27}$

The connection between the time-dependent correlation functions and the susceptibility is established by the Kubo formula for the response function

$$
\tilde{\chi}_{n}^{\xi \xi}(t)=i \Theta(t)\left\langle\left[S_{\delta}^{\xi}(t), S_{n}^{\xi}\right]\right\rangle, \quad \xi=x, y, z
$$

via the relation

$$
\chi_{\xi \xi}(q)=\sum_{n=-\infty}^{+\infty} e^{-i q n} \int_{-\infty}^{+\infty} d t \tilde{\chi}_{n}^{\xi \xi}(t)
$$

It follows that one can express the susceptibility $\chi_{\xi \xi}(q)$ in terms of the two-spin correlation functions (2.1) as

$$
\chi_{\xi \xi}(q)=\frac{i}{4} \sum_{n=-\infty}^{+\infty} e^{-i q n} \int_{-\infty}^{+\infty} d t \Xi_{n}(t) \operatorname{sgn}(t)
$$

or in terms of the dynamic structure factor

$$
S_{\xi \xi}(q, \omega) \equiv \frac{1}{4} \sum_{n=-\infty}^{+\infty} e^{-i q n} \int_{-\infty}^{+\infty} d t e^{i \omega t} \Xi_{n}(t)
$$

as

$$
\chi_{\xi \xi}(q)=\frac{1}{\pi} \int_{-\infty}^{+\infty} \frac{d \omega}{\omega} S_{\xi \xi}(q, \omega) .
$$

By using the identity

$$
\left[e^{-\beta H}, S_{n}^{\xi}\right]=\int_{0}^{\beta} d \tau e^{(\tau-\beta) H}\left[S_{n}^{\xi}, H\right] e^{-\tau H},
$$

one can write the formula (2.3) with (2.2) as

$$
\chi_{\xi \xi}(q)=\frac{1}{4} \sum_{n=-\infty}^{+\infty} e^{-i q n} \int_{0}^{\beta} d \tau \Xi_{n}(-i \tau)
$$

where $\beta=\left(k_{B} T\right)^{-1}$. If this formula is to be used at zero temperature $(\beta=\infty)$, great care must be exercised: timedependent two-point correlation functions of quantum systems have an inversion symmetry about the point $-i \beta / 2$ in the complex $t$ plane, characterized in our case by the condition ${ }^{30}$.

$$
\Xi_{n}(-t)=\Xi_{n}(t-i \beta) \text {. }
$$

This inversion symmetry of $\Xi_{n}(t)$ is lost at $T=0$, with the consequence that

$$
\lim _{\beta \rightarrow \infty} \lim _{\tau \rightarrow \beta} \Xi_{n}(-i \tau) \neq \lim _{\tau \rightarrow \infty} \lim _{\beta \rightarrow \infty} \Xi_{n}(-i \tau) .
$$

Thus, in Eq. (2.8) the integral has to be performed before the limit $\beta \rightarrow \infty$ is taken. However, by using (2.9), one can rewrite $(2.8)$ in the form

$$
\chi_{\xi \xi}(q)=\frac{1}{2} \sum_{n=-\infty}^{+\infty} e^{-i q n} \int_{0}^{\beta / 2} d \tau \Xi_{n}(-i \tau)
$$

In this formula, the limit $\beta \rightarrow \infty$ can be performed before the evaluation of the integral, since

$$
\lim _{\beta \rightarrow \infty} \lim _{t \rightarrow \beta / 2} \Xi_{n}(-i \tau)=\lim _{\tau \rightarrow \infty} \lim _{\beta \rightarrow \infty} \Xi_{n}(-i \tau) \text {. }
$$

Alternatively, one can derive Eq. (2.11) with $\beta \rightarrow \infty$ by the use of contour integrals starting from Eq. (2.4). ${ }^{31}$

It must be emphasized that the formal expression (2.11) with $\beta \rightarrow \infty$ is only useful if $\Xi_{n}(-i \tau)$ vanishes rapidly enough for $\tau \rightarrow \infty$ that the integral exists. If this is not the case, the complete sum, or a partial sum, over $n$ must be executed first, in order to render the integral finite. A similar comment applies to Eq. (2.4). In our case of the staggered susceptibilities $\bar{\chi}_{x x}$ of the TI and the $X Y$ models, we shall find it to be necessary to perform a pairwise summation under the integral in order to avoid divergences. We shall evaluate $\bar{\chi}_{x x}$ as the sum

$$
\bar{\chi}_{x x}=\sum_{n=1}^{\infty}(-1)^{n} C_{x x}(n),
$$

where

$$
C_{x x}(n)=\frac{1}{2} \int_{0}^{\infty} d \tau\left[X_{n}(-i \tau)-X_{n-1}(-i \tau)\right] .
$$

The function $C_{x x}(n)$ is finite for all $n$, and the series (2.13a) is convergent.

In the following, we shall derive a large- $n$ asymptotic 
expansion for the functions $\left[C_{x x}(n)\right]_{\mathrm{TI}}$ and $\left[C_{x x}(n)\right]_{X Y}$. For small $n$, where such an expansion is necessarily inadequate, the values of these functions are determined by a high-precision numerical calculation. The numerical value of the staggered susceptibility $\bar{\chi}_{x x}$ can then be determined very accurately from (2.11a).

Our energy unit throughout this paper is the exchange constant $J$. Therefore, the time variable and all susceptibilities are measured in units of $J^{-1}$. For reasons of notational simplicity, we set $J=1$ in the following.

\section{B. The calculation of $\left(\bar{\chi}_{x x}\right)_{T I}$}

As was shown in Ref. 24, the time-dependent correlation function $\left[X_{n}(t)\right]_{\mathrm{TI}}$ at $T=0$ can be expressed in terms of a related complex function $\sigma_{n}(z)$ as

$$
\left[X_{n}(t)\right]_{\mathrm{TI}}=\left[X_{n}(0)\right]_{\mathrm{TI}} \exp \left(-\frac{1}{2} t^{2}+\int_{0}^{2 t} d t^{\prime} \frac{\sigma_{n}\left(i t^{\prime}\right)}{t^{\prime}}\right),
$$

where the equal-time correlation function is given by $\left[X_{0}(0)\right]_{\mathrm{TI}}=1$ for $n=0$ and

$\left[X_{n}(0)\right]_{\mathrm{TI}}=\left(\frac{2}{\pi}\right)^{|n|} \prod_{l=1}^{|n|}\left[1-\frac{1}{4 l^{2}}\right)^{l-|n|}$ for $n \neq 0$.

The function $\sigma_{n}(z)$ satisfies the nonlinear ordinary differential equation (ODE)

$$
\left(z \sigma_{n}^{\prime \prime}\right)^{2}+4\left(z \sigma_{n}^{\prime}-\sigma_{n}-n^{2}\right)\left[z \sigma_{n}^{\prime}-\sigma_{n}+\left(\sigma_{n}^{\prime}\right)^{2}\right]=0
$$

with initial conditions to be given below.

A major part of the present project is the solution to this ODE for general $n$, yielding $\left[X_{n}(t)\right]_{\mathrm{TI}}$ and, by a functional relation, $\left[X_{n}(t)\right]_{X Y}$ which are then used in Eq. (2.13) to calculate $\left(\bar{\chi}_{x x}\right)_{\text {TI }}$ and $\left(\bar{\chi}_{x x}\right)_{X Y}$. If it were possible, one would use initial conditions for $\sigma_{n}(z)$ and $\sigma_{n}^{\prime}(z)$ at $z=0$, since this is the starting point in the integration of the ODE (2.16) for use in (2.14). However, the (regular) singular point in (2.16) at $z=0$ renders this impossible, and instead, one uses a series expansion for $\sigma_{n}(z)$, valid near $z=0$, and the resultant one for $\sigma_{n}^{\prime}(z)$, as initial conditions:

$$
\sigma_{n}(z)=\sum_{j=1}^{\infty} \xi_{j}^{(n)} z^{j}
$$

The general form and special values of the coefficients $\xi_{j}^{(0)}$ for the special case $n=0$ were determined in Ref. 24. For $n \geq 1$ we find that the coefficients for even and odd powers have the following general forms, respectively:

$$
\begin{aligned}
& \xi_{2 k}^{(n)}=\sum_{l=0}^{\frac{k}{2 n+1} c_{2 k, 2 l}^{(n)} \pi^{-2 l}} \\
& \xi_{2 k+1}^{(n)}=\left\{\begin{array}{l}
0, k<n \\
\pi^{-1}\left[\frac{k}{2 n+1}\right] \\
\sum_{l=0}^{0} c_{2 k+1,2 l+1} \pi^{-2 l}, k \geq n
\end{array}\right.
\end{aligned}
$$

where $[v]$ denotes the integral part of $v$. The coefficient of the lowest odd power in the series is given by

$$
\xi_{2 n+1}^{(n)}=\pi^{-1} c_{2 n+1,1}^{(n)}=\left\{\begin{array}{l}
-\pi^{-1}, \quad n=0 \\
(-1)^{n-1} \pi^{-1} \prod_{l=1}^{n}\left[\frac{l}{2(2 l-1)^{2}(2 l+1)}\right], n \geq 1 .
\end{array}\right.
$$

The coefficients for all higher odd powers in (2.17) can be calculated recursively in terms of $\xi_{2 n+1}^{(n)}$. For the even coefficients we have found that $c_{2 k, 0}^{(n)}$, in particular, have the general form

$$
c_{2 k, 0}^{(n)}=\frac{n^{2} P_{2 k}^{(n)}}{\prod_{l=0}^{k-1}\left[4 n^{2}-(2 l+1)^{2}\right]^{v_{k, l}}},
$$

where

$$
v_{k, l}=1+\left[\frac{2 k-1}{4 l+2}\right]
$$

$P_{2 k}^{(n)}$ is a polynomial in $n^{2}$, and the bracket in (2.20b) denotes an integral part. Specifically, for $k=1, \ldots, 5$,

$$
\begin{aligned}
& P_{2}^{(n)}=-1, \\
& P_{4}^{(n)}=1,
\end{aligned}
$$

$$
\begin{aligned}
& P_{6}^{(n)}=2\left(2 n^{2}+1\right) \\
& P_{8}^{(n)}=64 n^{6}-32 n^{4}-392 n^{2}-45 \\
& P_{10}^{(n)}=2\left(-128 n^{8}-224 n^{6}+3160 n^{4}+1854 n^{2}+63\right) .
\end{aligned}
$$

Coefficients equivalent to $c_{2,0}^{(n)}$ and $c_{4,0}^{(n)}$ were given previously; ${ }^{24}$ the general formula (2.19) and the $c_{2 k, 0}^{(n)}$, for $6 \leq 2 k \leq 10$ are new here. The $c_{2 k, 2 l}^{(n)}$ with $l \neq 0$ can be calculated recursively in terms of lower-order coefficients.

The small- $z$ expansion for $n=0$ was used in Ref. 24 for the numerical solution of the ODE from which the longtime asymptotic expansion was inferred. For the present work the $\xi_{j}^{(n)}$ for $n \leq 10$ and $j \leq 20$ have been computed. As is discussed below, the expansions for $n \leq 5$ are used for the numerical solutions of the respective cases of the ODE (2.16). For reasons of space we list the $c_{j, k}^{(n)}$ only for $1 \leq n \leq 2$ in the Appendix. 
We proceed to consider the asymptotic behavior of $\left[X_{n}(t)\right]_{\mathrm{TI}}$ for $n, t \rightarrow \infty$. First, we recall that since timedependent correlation functions have the general properties $\Xi_{n}(t)=\Xi_{-n}(t)$ and $\Xi_{n}(t)=\Xi_{n}(-t)^{*}$, one does not incur any loss of generality by taking $n>0$ and, for the case of real $t, t>0$. For $n, t \rightarrow \infty$, it was found in Ref. 25 that one has to distinguish three regimes: (1) the "timelike" regime where $0 \leq n / t<1$; (2) the "space-like" regime where $0 \leq t / n<1$; and (3) a crossover regime where $t=n+n^{1 / 3} x$ with $x=O(1)$ fixed. The asymptotic expansions (AE's) of $\left[X_{n}(t)\right]_{\mathrm{TI}}$ have different structures in different regimes.

For imaginary times, $t=-i \tau, \tau>0$, both $\sigma_{n}$ and $X_{n}$ are real functions and, most importantly, there is only a single regime in the corresponding $\mathrm{AE}$ for $\tau, n \rightarrow \infty$, which corresponds to the space-like regime for real $t$. For our purpose of calculating the staggered susceptibility, we use the following result ${ }^{25}$ for the AE of $\left[X_{n}(-i \tau)\right]_{\mathrm{TI}}$ :

$$
\begin{aligned}
{\left[X_{n}(-i \tau)\right]_{\mathrm{TI}} \sim \bar{A}\left(n^{2}+\tau^{2}\right)^{-1 / 8}[} & 1+\frac{1}{2^{6}}\left(n^{2}+\tau^{2}\right)^{-3}\left(2 \tau^{4}-5 \tau^{2} n^{2}-n^{4}\right) \\
& \left.+\frac{3}{2^{13}}\left(n^{2}+\tau^{2}\right)^{-6}\left(108 \tau^{8}-1500 \tau^{6} n^{2}+1127 \tau^{4} n^{4}+46 \tau^{2} n^{6}+11 n^{8}\right)+O\left([\max (n, \tau)]^{-6}\right)\right]
\end{aligned}
$$

where the constant $\bar{A}$ is given by

$$
\bar{A}=2^{1 / 12} e^{3 \xi^{\prime}(-1)}=0.645002448 \ldots .
$$

We expect that this asymptotic formula is very accurate for all $\tau>0$ if $n>n_{0}$ as well as for $\tau>\tau_{-}(n)$ if $n \leq n_{0}$, where $n_{0}$ and $\tau_{-}(n)$ have to be determined empirically for any required accuracy. For $\tau=0$ we can check the prediction of (2.18) against the exact result (2.14). We find agreement to within one part in $10^{-7}$ for $n$ as low as $n=5$.

We have solved the ODE (2.16) numerically for $n=0,1, \ldots, 5$ and generated the corresponding functions $\left[X_{n}(-i \tau)\right]_{\mathrm{TI}}$ for $0 \leq \tau \leq 20$. We find that for any given $n$ there exists a time interval $\tau_{-}(n)<\tau<\tau_{+}(n)$ within which our numerical result for $\left[X_{n}(-i \tau)\right]_{\mathrm{TI}}$ matches the $\mathrm{AE}$ (2.26) to within a relative error of $10^{-6}$. We have then determined the values of $\left[C_{x x}(n)\right]_{\mathrm{TI}}$ for $n=1,2, \ldots, n_{0}$ where $n_{0}=5$ by integrating the numerically generated functions $\left[X_{n}(-i \tau)\right]_{\mathrm{TI}}$ from $\tau=0$ to $\tau_{0}(n)=\frac{1}{2}\left[\tau_{+}(n)\right.$ $\left.-\tau_{-}(n)\right]$ and by integrating the corresponding AE's from $\tau=\tau_{0}$ to $\tau=\infty$. The five values $\left[C_{x x}(n)\right]_{\mathrm{TI}}, n=1, \ldots, 5$, thus computed, for which we obtain a relative accuracy of $\sim 10^{-6}$, are listed in Table I.

We next proceed to derive a large- $n$ asymptotic expansion for the function $\left[C_{x x}(n)\right]_{\mathrm{TI}}$ from (2.13b) and (2.26). We find that it has the structure

$$
\left[C_{x x}^{(\mathrm{AE})}(n)\right]_{\mathrm{TI}} \sim-B_{\mathrm{TI}} \sum_{m=0}^{\infty} a_{m} n^{-(m+1 / 4)},
$$

with

TABLE I. The first five values of the function $\left[C_{x x}(n)\right]_{\mathrm{TI}}$ as obtained from Eq. (2.13) by numerical integration and the values of its asymptotic expansion $\left[C_{x x}^{(\mathrm{AE})}(n)\right]_{\mathrm{TI}}$ given in Eq. (2.28).

\begin{tabular}{lcc}
\hline \hline$n$ & $-\left[C_{x x}(n)\right]_{\mathrm{TI}}$ & $-\left[C_{x x}^{(\mathrm{AE})}(n)\right]_{\mathrm{TI}}$ \\
\hline 1 & 0.122906 & 0.122735 \\
2 & 0.097484 & 0.097479 \\
3 & 0.086264 & 0.086264 \\
4 & 0.079434 & 0.079434 \\
5 & 0.074648 & 0.074648 \\
\hline \hline
\end{tabular}

$$
B_{\mathrm{TI}}=\frac{1}{2} \bar{A} \pi^{1 / 2} \Gamma\left(\frac{5}{8}\right) / \Gamma\left(\frac{1}{8}\right) .
$$

The first six coefficients $a_{m}$, which can be derived from (2.26) to the given order, are listed in Table II. The values of $\left[C_{x x}^{(\mathrm{AE})}(n)\right]_{\mathrm{TI}}$ for $n=1,2, \ldots, n_{0}$ obtained from these six terms are included in Table $I$. We can expect that the $\mathrm{AE}$ of $\left[C_{x x}(n)\right]_{\mathrm{TI}}$ converges about as rapidly as the $\mathrm{AE}$ of $\left[X_{n}(0)\right]_{\mathrm{TI}}$ noted above. Comparison with the numerical results shows that this is indeed the case: For the required accuracy, the AE (2.28) can be used in (2.13a) for all $n \geq n_{0}$. We thus rewrite (2.13a) in the form

$$
\left(\bar{\chi}_{x x}\right)_{\mathrm{TI}} \simeq \sum_{n=1}^{n_{0}}(-1)^{n}\left[C_{x x}(n)\right]_{\mathrm{TI}}+\left(V_{x x}\right)_{\mathrm{TI}},
$$

where the values of $\left[C_{x x}(n)\right]_{\mathrm{TI}}$ are given in Table $\mathrm{I}$ and

$$
\left(V_{x x}\right)_{\mathrm{TI}} \simeq \sum_{n=n_{0}+1}^{\infty}(-1)^{n}\left[C_{x x}^{(\mathrm{AE})}(n)\right]_{\mathrm{TI}}
$$

The infinite sum over $n$ in (2.31) for each individual term of $\left[C_{x x}^{(\mathrm{AE})}(n)\right]_{\mathrm{TI}}$, as given in (2.28), can be expressed in terms of "incomplete" $\eta$ functions defined as

$$
\eta(z, l)=\sum_{k=l+1}^{\infty}(-1)^{k-1} k^{-z}
$$

This function is related to the familiar Riemann $\zeta$ function according to

TABLE II. The first six coefficients $a_{m}$ of the function [ $\left.C_{x x}(n)\right]_{\mathrm{TI}}$ and the values of the corresponding incomplete $\eta$ functions necessary to evaluate $\left(V_{x x}\right)_{T I}$.

\begin{tabular}{lcc}
\hline \hline$m$ & $a_{m}$ & $\eta\left(m+\frac{1}{4}, 5\right)$ \\
\hline 0 & 1 & -0.326085408 \\
1 & $\frac{1}{8}$ & -0.058702956 \\
2 & $\frac{55}{3264}$ & -0.010489118 \\
3 & $-\frac{45}{4352}$ & -0.001861091 \\
4 & $-\frac{452205}{62808064}$ & -0.000328064 \\
5 & $\frac{98917}{29556736}$ & -0.000057482 \\
\hline \hline
\end{tabular}




$$
\eta(z, 0) \equiv \eta(z)=\left(1-2^{1-z}\right) \zeta(z)
$$

Hence,

$$
\left(V_{x x}\right)_{\mathrm{TI}} \simeq B_{\mathrm{TI}} \sum_{m=0}^{m_{0}} a_{m} \eta\left(m+1 / 4, n_{0}\right)
$$

with $m_{0}=5$ according to the maximum number of known expansion coefficients, given in Table II. Also listed in Table II are the corresponding values of the function $\eta\left(m+1 / 4, n_{0}\right)$ for $0 \leq m \leq m_{0}$.

In (2.30), (2.31), and (2.34), we have replaced the equality symbol by $\simeq$ in order to remind the reader that the right-hand sides of these equations are obtained by summing up terms of an asymptotic series. For a given $n_{0}$ this series would not converge if $m_{0} \rightarrow \infty$. Therefore, if one wants to increase the accuracy of the results, it is necessary to increase both $m_{0}$ and $n_{0}$, as well as $\tau_{0}(n)$, in the calculation. This, in turn, requires that one calculate $X_{n}(-i \tau)$ from the ODE over a larger portion of $(n, \tau)$ space with increased accuracy. It is here that one reaches a limiting accuracy. The evaluation of (2.30) with (2.34) and the numbers of Tables I and II yield the following numerical value for the $T=0$ staggered susceptibility $\bar{\chi}_{x x}$ of the TI model:

$$
\left(\bar{\chi}_{x x}\right)_{\mathrm{TI}}=0.070593 \pm 0.000005
$$

\section{The calculation of $\left(\bar{\chi}_{x x}\right)_{X Y}$}

We can also determine the staggered susceptibility $\left(\bar{\chi}_{x x}\right)_{X Y}=\left(\bar{\chi}_{y y}\right)_{X Y}$ of the $X Y$ model at $T=0$ from our results for the TI model discussed above, by employing the relation ${ }^{24}$

$$
X_{n}(t)_{X Y}=Y_{n}(t)_{X Y}=\left\{\begin{array}{l}
{\left[X_{n / 2}(t / 2)\right]_{\mathrm{TI}}\left[X_{n / 2}(t / 2)\right]_{\mathrm{TI}}, n \text { even }} \\
{\left[X_{(n-1) / 2}(t / 2)\right]_{\mathrm{TI}}\left[X_{(n+1) / 2}(t / 2)\right]_{\mathrm{TI}}, n \text { odd }}
\end{array}\right.
$$

between the corresponding time-dependent correlation functions. The same data which have been used to calculate the values $\left[C_{x x}(n)\right]_{\mathrm{TI}}$ for $n=1,2, \ldots, n_{0}$ according to $(2.13 \mathrm{~b})$ can be used, with $(2.36)$, to calculate, with equal accuracy, the values $\left[C_{x x}(n)\right]_{X Y}$ for $n=1,2, \ldots, 2 n_{0}$. These values are listed in Table III.

In Ref. 25 the relation (2.36) was used to derive an asymptotic expansion for $\left[X_{n}(t)\right]_{X Y}$ from the AE of $\left[X_{n}(t)\right]_{\mathrm{TI}}$. For negative imaginary $t$ it has the following structure:

$$
\begin{aligned}
X_{n}(-i \tau)_{X Y} \sim 2^{1 / 2}(\bar{A})^{2}\left(n^{2}+\tau^{2}\right)^{-1 / 4} & \left(1+2^{-2} k_{n}\left(n^{2}+\tau^{2}\right)^{-2}\left(n^{2}-\tau^{2}\right)+2^{-3}\left(n^{2}+\tau^{2}\right)^{-3}\left(-n^{4}-5 n^{2} \tau^{2}+2 \tau^{4}\right)\right. \\
& +2^{-5} k_{n}\left(n^{2}+\tau^{2}\right)^{-5}\left(-8 n^{6}-165 n^{4} \tau^{2}+390 n^{2} \tau^{4}-41 \tau^{6}\right) \\
& +2^{-7}\left(n^{2}+t^{2}\right)^{-6}\left(17 n^{8}+74 n^{6} \tau^{2}+1701 n^{4} \tau^{4}\right. \\
- & \left.\left.-2260 n^{2} \tau^{6}+164 \tau^{8}\right)+O\left([\max (\tau, n)]^{-6}\right)\right\}
\end{aligned}
$$

with $k_{n}=\frac{1}{2}\left[1-(-1)^{n}\right]$ and $\bar{A}$ as given in (2.27). From (2.13b) with (2.37) we derive an $\mathrm{AE}$ for the function $\left[C_{x x}(n)\right]_{X Y}$ of the form

$$
C_{x x}^{(\mathrm{AE})}(n)_{X Y} \sim-B_{X Y} \sum_{m=0}^{\infty}\left[b_{m}+(-1)^{n} c_{m}\right] n^{-(m+1 / 2)},
$$

with

$$
B_{X Y}=2^{-1 / 2} \pi^{1 / 2}(\bar{A})^{2} \Gamma\left(\frac{3}{4}\right) / \Gamma\left(\frac{1}{4}\right) .
$$

Because of the alternating character of some of the terms in (2.37), the structure of (2.38) is more complicated than that of (2.28). The first five coefficients $b_{m}$ and $c_{m}$, which can be inferred from (2.37), are listed in Table IV. The values of $\left[C_{x x}^{(\mathrm{AE})}(n)\right]_{X Y}$ for $n=1,2, \ldots, 2 n_{0}$ obtained from these five terms are included in Table III for comparison with the numerical results for $\left[C_{x x}(n)\right]_{X Y}$. We observe that the deviation between the two functions drops to values below one part in $10^{-6}$ before $n$ reaches $2 n_{0}$. Hence, we use the AE (2.38) in (2.13a) for all $n \gtrsim 2 n_{0}$.

In analogy to the TI case we rewrite the staggered susceptibility as

$$
\left(\bar{\chi}_{x x}\right)_{X Y} \simeq \sum_{n=1}^{2 n_{0}}(-1)^{n}\left[C_{x x}(n)\right]_{X Y}+\left(V_{x x}\right)_{X Y},
$$

with the values of $\left[C_{x x}(n)\right]_{X Y}$ given in Table III and

$$
\left(V_{x x}\right)_{X Y} \simeq \sum_{n=2 n_{0}+1}^{\infty}(-1)^{n}\left[C_{x x}^{(\mathrm{AE})}(n)\right]_{X Y}
$$

TABLE III. The first ten values of the function $\left[C_{x x}(n)\right]_{X Y}$ as obtained from Eq. (2.13) by numerical integration and the values of its asymptotic expansion $\left[C_{x x}^{(\mathrm{AE})}(n)\right]_{X Y}$ given in Eq. (2.38).

\begin{tabular}{rcc}
\hline \hline$n$ & $-\left[C_{x x}(n)\right]_{X Y}$ & $-\left[C_{x x}^{(\mathrm{AE})}(n)\right]_{X Y}$ \\
\hline 1 & 0.185463 & 0.191917 \\
2 & 0.145678 & 0.144826 \\
3 & 0.105571 & 0.105566 \\
4 & 0.095884 & 0.095863 \\
5 & 0.080831 & 0.080830 \\
6 & 0.076191 & 0.076189 \\
7 & 0.067890 & 0.067890 \\
8 & 0.065064 & 0.065063 \\
9 & 0.059646 & 0.059646 \\
10 & 0.057699 & 0.057699 \\
\hline \hline
\end{tabular}


TABLE IV. The first five coefficients $b_{m}$ and $c_{m}$ of the asymptotic expansion Eq. (2.38) of the function $\left[C_{x x}(n)\right]_{X Y}$ and the values of the corresponding incomplete $\eta$ functions necessary to evaluate $\left(V_{x x}\right)_{X Y}$.

\begin{tabular}{ccccc}
\hline \hline$m$ & $b_{m}$ & $\eta\left(m+\frac{1}{2}, 10\right)$ & $c_{m}$ & $\zeta\left(m+\frac{1}{2}, 10\right)$ \\
\hline 0 & 1 & 0.154173201 & 0 & \\
1 & $\frac{1}{4}$ & 0.014633977 & $\frac{1}{10}$ & 0.617038855 \\
2 & $-\frac{1}{40}$ & 0.001386003 & $\frac{3}{40}$ & 0.019566422 \\
3 & $-\frac{7}{64}$ & 0.000130989 & $-\frac{245}{7072}$ & 0.001115983 \\
4 & $-\frac{7}{4420}$ & 0.000012354 & $-\frac{1631}{14144}$ & 0.000075718 \\
\hline
\end{tabular}

In this case the sum over $n$ for each individual term of $\left[C_{x x}^{(\mathrm{AE})}(n)\right]_{X Y}$ can be expressed in terms of the "incomplete" $\eta$ functions (2.32) and of correspondingly defined incomplete $\zeta$ functions

$$
\zeta(z, l) \equiv \sum_{k=l+1}^{\infty} k^{-z},
$$

yielding

$$
\begin{aligned}
\left(V_{x x}\right)_{X Y} \simeq B_{X Y} \sum_{m=0}^{m_{0}}\left[b_{m} \eta\left(m+\frac{1}{2}, 2 n_{0}\right)\right. \\
\left.-c_{m} \xi\left(m+\frac{1}{2}, 2 n_{0}\right)\right],
\end{aligned}
$$

with $n_{0}=5$ as in the TI case. The coefficients $b_{m}$ and $c_{m}$ are known up to $m_{0}=4$. They are listed in Table IV along with the values of the corresponding incomplete $\eta$ functions and $\xi$ functions. The $T=0$ staggered susceptibility $\left(\bar{\chi}_{x x}\right)_{X Y}$ is then obtained from (2.40) with (2.43) and the numbers from Tables III and IV. The result is

$$
\left(\bar{\chi}_{x x}\right)_{X Y}=0.075566 \pm 0.000005 \text {. }
$$

\section{THE DIVERGENCE IN $\chi_{x x}(q)$ AT $T=0$}

A straightforward generalization of our calculational scheme for the $T=0$ staggered susceptibilities $\bar{\chi}_{x x}=\chi_{x x}(q=\pi)$ is possible only for special $q$ values of the form $q=m \pi / M$, where $m$ and $M$ are integers with no common divisor and $m$ is odd. Then the $q$-dependent susceptibility can be written in the form

$$
\begin{aligned}
& \chi_{x x}\left(\frac{m \pi}{M}\right)=-\sum_{n=1}^{\infty} \cos \left[(n-1) \frac{m \pi}{M}\right] C_{x x}(n, M), \\
&-\sum_{n=1}^{[M / 2]} \cos \left[\frac{n m \pi}{M}\right] C_{x x}(n+1, M-2 n), \\
& C_{x x}(n, M)=\frac{1}{2} \int_{0}^{\infty} d \tau\left[X_{n-1+M}(-i \tau)-X_{n-1}(-i \tau)\right],
\end{aligned}
$$

where $[M / 2]$ denotes the integral part of $M / 2$, all $C_{x x}(n, M)$ are finite and the series converges. ${ }^{32}$ Thus, for each $M$ a new large- $n$ AE for the function $C_{x x}(n, M)$ must be calculated and the numerical values for small $n$ must be determined. We have executed this calculation only for $m=M=1(q=\pi)$, as described in Sec. II. For general $q$, i.e., such that $q / \pi$ is not rational, the complete sum over $n$ in (2.11) has to be performed first because of the necessity of canceling divergences which would be present in a partially summed result. This yields the $q$ dependent correlation function

$$
G_{x x}(q,-i \tau)=\sum_{n=-\infty}^{+\infty} e^{-i q n} X_{n}(-i \tau)
$$

from which the susceptibility is then calculated as

$$
\chi_{x x}(q)=\frac{1}{2} \int_{0}^{\infty} d \tau G_{x x}(q,-i \tau) .
$$

The integral (3.3) diverges only for $q \rightarrow 0$. Whereas the value of $\chi_{x x}(q)$ for arbitrary $q$ depends on the full details of the correlation function $X_{n}(-i \tau)$ for all $n$ and $\tau$, as we have seen in Sec. II, the nature of the divergence in $\chi_{x x}(q)$ for $q \rightarrow 0$ depends only on the function $X_{n}(-i \tau)$ for large $n$ but all $\tau>0$. Hence it can be determined from the AE alone. In fact, only the leading terms

$$
X_{n}(-i \tau) \sim\left\{\begin{array}{l}
\bar{A}\left(n^{2}+\tau^{2}\right)^{-1 / 8} \quad(\mathrm{TI}), \\
2^{1 / 2}(\bar{A})^{2}\left(n^{2}+\tau^{2}\right)^{-1 / 4}
\end{array}(X Y)\right.
$$

contribute to the divergence in $\chi_{x x}(q)$, yielding the following results for the TI and $X Y$ models, respectively: ${ }^{39}$

$$
\chi_{x x}(q) \sim\left\{\begin{array}{l}
2^{-1 / 4} \bar{A} \cos (3 \pi / 8) \Gamma^{2}\left(\frac{7}{8}\right) q^{-7 / 4} \quad(\mathrm{TI}), \\
2^{-1 / 2}(\bar{A})^{2} \Gamma^{2}\left(\frac{3}{4}\right) q^{-3 / 2}(X Y)
\end{array}\right.
$$

These results on the divergent susceptibilities are compatible with the form

$$
S_{x x}(q, \omega) \sim a\left(\omega^{2}-q^{2}\right)^{-\alpha} \Theta(\omega-q)
$$

for the dominant singularity in the dynamic structure factor for $\omega, q \rightarrow 0$. By using the ansatz (3.6) in (2.6) and comparing the result with (3.5), we can unambiguously determine the exponent $\alpha$ and the amplitude $a$ in (3.5):

$$
\begin{aligned}
& \alpha_{\mathrm{TI}}=\frac{7}{8}, \quad a_{\mathrm{TI}}=2^{3 / 4} \bar{A} \pi \cos (3 \pi / 8) \Gamma\left(\frac{7}{8}\right) / \Gamma\left(\frac{1}{8}\right), \\
& \alpha_{X Y}=\frac{3}{4}, \quad a_{X Y}=2^{1 / 2}(\bar{A})^{2} \pi \Gamma\left(\frac{3}{4}\right) / \Gamma\left(\frac{1}{4}\right) .
\end{aligned}
$$

It is interesting that the parameters (3.7) are also obtained from the exactly known divergences in the integrated intensities 


$$
\begin{aligned}
I_{x x}(q) & \equiv \sum_{n=-\infty}^{\infty} e^{-i q n} X_{n}(0) \\
& \sim\left\{\begin{array}{l}
\frac{1}{2} \bar{A} \Gamma\left(\frac{3}{4}\right) \cos (3 \pi / 8) q^{-3 / 4} \\
\frac{1}{2}(\bar{A})^{2} \Gamma\left(\frac{1}{2}\right) q^{-1 / 2},
\end{array}\right.
\end{aligned}
$$

by using

$$
I_{x x}(q)=\int_{0}^{\infty} \frac{d \omega}{2 \pi} S_{x x}(q, \omega)
$$

with the ansatz (3.6).

The form (3.6) for the $X Y$ model with the correct exponent $\alpha_{X Y}=\frac{3}{4}$ was first obtained by Luther and Peschel $^{34}$ on the basis of their calculation in the framework of the continuum approximation (Luttinger model). In that approach, the amplitude $a_{X Y}$ is a cutoff dependent quantity and can therefore not be determined. More recently, an analytic expression for $S_{x x}(q, \omega)$ of the $X Y$ model was conjectured for wave numbers throughout the Brillouin zone and frequencies up to $2 J$, thus covering the contributions of two excitation continua. ${ }^{22}$ For $q, \omega \rightarrow 0$, this expression can be reduced to the form (3.6) with the correct $\alpha_{X Y}=\frac{3}{4}$ and the amplitude

$a_{X Y}=2^{3 / 2} \pi^{1 / 2}(1+2 / \pi) / \Gamma^{2}\left(\frac{1}{4}\right)=0.6241 \ldots$.

Although (3.10) does not involve the constant $\bar{A}$ and is thus distinctively different from the exact value $a_{X Y}=0.6247 \ldots$, it is astonishingly close, numerically.

\section{DISCUSSION}

Let us now discuss our new results presented in Secs. II and III in the context of existing susceptibility results for the two models characterized by the Hamiltonians (1.4) and (1.5), respectively. We shall restrict our discussion to $T=0$ results.

\section{A. The anisotropic $X Y$ model $H_{A-X Y}$}

The anisotropic $X Y$ model (1.4) with $\gamma \neq 0$ is well known to exhibit at $h=h_{c}=1$, a second-order phase transition from a phase with spontaneous magnetization $M_{\boldsymbol{x}}$ at $h<1$ to a phase without spontaneous long-range order at $h>10^{3,7}$ The direct susceptibility $\chi_{z z}$ is known for all $\gamma$ and $h ;^{3,5,14,19}$ it has a logarithmic divergence at $h=h_{c}$. This divergence is related to the logarithmic divergence in the 2D Ising model specific heat as a function of temperature. ${ }^{9}$ At $h=h_{c}, \chi_{z z}(q)$ diverges logarithmically as $q \rightarrow 0$. For the special case $h=\gamma=1$, which is the TI model, the result is

$$
\begin{aligned}
{\left[\chi_{\mathrm{zz}}(q)\right]_{\mathrm{TI}}=} & \frac{1}{4 \pi \sin (q / 4)} \ln \left[\frac{1+\sin (q / 4)}{1-\sin (q / 4)}\right) \\
& +\frac{1}{4 \pi \cos (q / 4)} \ln \left(\frac{1+\cos (q / 4)}{1-\cos (q / 4)}\right)-\frac{1}{\pi} .
\end{aligned}
$$

The staggered susceptibility $\bar{\chi}_{z z}$, on the other hand, is finite for all $\gamma \neq 0$ and $h$. It diverges only for $\gamma \rightarrow 0, h \rightarrow 0$. At $\gamma=h=0$, which corresponds to the isotropic $X Y$ model, the $q$-dependent susceptibility $\chi_{z z}(q)$ diverges logarithmically as $q \rightarrow \pi$ :

$$
\left[\chi_{z z}(q)\right]_{X Y}=\frac{1}{2 \pi \sin (q / 2)} \ln \left(\frac{1+\sin (q / 2)}{1-\sin (q / 2)}\right) .
$$

Interesting behavior is displayed by the function $\chi_{z z}(q)$ at $\gamma=0$ and $0 \leq h \leq 1$ : The logarithmic divergence moves from the Brillouin-zone boundary towards the zone center as the field is increased. The divergence occurs at the special wave number $q_{s}=\pi\left(1-2 M_{z}\right)$ with $M_{z}$ $=\pi^{-1} \arcsin (h)$, for which the excitation spectrum is gapless. ${ }^{35}$

The susceptibility $\chi_{y y}(q)$ is, in general, not known, except for $\gamma=1$. For this case, van Dongen and Capel ${ }^{19}$ showed that

$$
\chi_{y y}(q)=M_{z} / h
$$

independent of $q$, where the magnetization function $M_{z}(h)$ is well known. For the TI case $(h=1)$, this remarkably simple result can easily be reproduced in the framework of the approach employed in Sec. II as follows:

$$
\begin{aligned}
{\left[\chi_{y y}(q)\right]_{\mathrm{TI}} } & =\frac{1}{2} \sum_{n=-\infty}^{+\infty} e^{-i q n} \int_{0}^{\infty} d \tau \frac{d^{2}}{d \tau^{2}} X_{n}(-i \tau) \\
& =-\left.\frac{1}{2} \sum_{n} e^{-i q n} \frac{d}{d \tau} X_{n}(-i \tau)\right|_{\tau=0}=\frac{1}{\pi}
\end{aligned}
$$

Here we have used, apart from (2.11), the fact that $\left[Y_{n}(t)\right]_{\mathrm{TI}}$ can be expressed as ${ }^{11}$

$$
\left[Y_{n}(t)\right]_{\mathrm{TI}}=-\frac{d^{2}}{d t^{2}}\left[X_{n}(t)\right]_{\mathrm{TI}},
$$

the known initial slope of the function $X_{n}(t):{ }^{24}$

$$
\frac{d}{d t}\left[X_{n}(t)\right]_{\mathrm{TI}}=-2 i M_{z} \delta_{n, 0}
$$

and the value $M_{z}=1 / \pi$ for the magnetization at the critical field. Thus the special property (4.5) combined with the special initial conditions (4.6) produce the striking effect that $\left[\chi_{y y}(q)\right]_{\mathrm{TI}}$ is $q$ independent and involves only a static thermodynamic quantity, viz., $M_{Z}$. This is in contrast to both $\left[\chi_{x x}(q)\right]_{\mathrm{TI}}$ and $\left[\chi_{\mathrm{zz}}(q)\right]_{\mathrm{TI}}$, which vary with $q$ and depend on the full details of their respective correlation functions $\left[X_{n}(t)\right]_{\mathrm{TI}}$ and $\left[Z_{n}(t)\right]_{\mathrm{TI}}$.

The susceptibility $\chi_{x x}(q)$ is the most difficult to evaluate even for $\gamma=1$; exact results are, therefore, scarce. We expect $\bar{\chi}_{x x}$ to stay finite for all $\gamma$ and $h$, and we know that $\chi_{x x}$ diverges at the critical field $h_{c}=1$. The nature of this divergence was determined on the basis of Suzuki's mapping of the anisotropic $X Y$ model onto the 2D Ising model as ${ }^{9}$

$$
\chi_{x x} \sim\left|h-h_{c}\right|^{-7 / 4} .
$$

According to our result (3.5a), the same power-law divergence characterizes the $q$-dependent susceptibility $\left[\chi_{x x}(q)\right]_{\mathrm{TI}}$ for $q \rightarrow 0$. The direct susceptibility $\chi_{x x}$ also diverges in the limit $\gamma \rightarrow 0$ for $0 \leq h \leq 1$, where the excitation gap again vanishes. For $\gamma=h=0$, this is reflected in 
our result (3.5b) for the $q$-dependent susceptibility $\left[\chi_{x x}(q)\right]_{X Y}$. This result for the divergence of $\left[\chi_{x x}(q)\right]_{\mathrm{XY}}$ can, in fact, be generalized to the case $\gamma=0,0 \leq h<1$ if the result

$$
X_{n}(-i \tau) \sim(\bar{A})^{2}(2 c)^{1 / 2}\left(n^{2}+c^{2} \tau^{2}\right)^{-1 / 4}, \quad c=\left(1-h^{2}\right)^{1 / 2}
$$

of Vaidya and Tracy ${ }^{28}$ is used for the leading term in the AE of $X_{n}(-i \tau)$, yielding

$$
\chi_{x x}(q) \sim(2 c)^{-1 / 2}(\bar{A})^{2} \Gamma^{2}\left(\frac{3}{4}\right) q^{-3 / 2} .
$$

As $h$ approaches the critical field, the amplitude in (4.9) diverges. This reflects the crossover from the $q^{-3 / 2}$ divergence to the $q^{-2}$ divergence at $h=1$ of the exact result

$$
\chi_{x x}(q)=[2(h-\cos q)]^{-1}
$$

which is valid for all $h \geq 1$. Our new results (2.35) and (2.44) for the staggered susceptibilities $\left[\left(\bar{\chi}_{x x}\right)\right]_{\mathrm{TI}}$ and $\left[\left(\bar{\chi}_{x x}\right)\right]_{X Y}$, respectively, are the only ones available that depend on the full details of the time-dependent correlation functions $X_{n}(t)$, whose structure was shown to be very complex. The $X Y$ value will be further discussed below in the context of the $X X Z$ model. For the TI case we thus know the numerical values of all three components of $\bar{\chi}_{\xi \xi}$ :

$$
\begin{aligned}
& \left(\bar{\chi}_{x x}\right)_{\mathrm{TI}}=0.07059 \ldots, \\
& \left(\bar{\chi}_{y y}\right)_{\mathrm{TI}}=1 / \pi=0.31830 \ldots, \\
& \left(\bar{\chi}_{z z}\right)_{\mathrm{TI}}=\pi^{-1}[\ln (1+\sqrt{2})-1]=0.07845 \ldots .
\end{aligned}
$$

We conclude by noting a striking similarity between the value (4.11a) and the value obtained by an approximate calculation of Tanaka and Ury $\hat{u}^{36}$ for the susceptibility of the isotropic square-lattice Ising antiferromagnet at $T=T_{c}$. The 1D $S=\frac{1}{2}$ transverse Ising model at the critical field can be related to a limiting case of the 2D Ising model at $T_{c} ;{ }^{9}$ however, the latter model is infinitely anisotropic with one exchange constant diverging and the other vanishing. Accordingly, it is not clear that our value (4.11a) should be equal to the result given in Eq. (5.2) of Ref. 36.

\section{B. The $X X Z$ model}

As noted in the introduction, the $X Y$ model (1.3), for which we have produced novel results, can also be regarded as the special case $\Delta=0$ of the interesting $X X Z$ model (1.5). For $\Delta>1$, the ground state of this model is ferromagnetic with all spins aligned parallel to the $z$ axis. The $T=0$ parallel $(z z)$ susceptibility vanishes identically and the perpendicular susceptibility $(x x=y y \equiv \perp 1)$ is exactly determined by the ferromagnetic spin-wave theory:

$$
\begin{aligned}
& \chi_{z z}(q)=0, \\
& \chi_{1 \perp}(q)=[2(\Delta-\cos q)]^{-1} .
\end{aligned}
$$

Hence, the direct perpendicular susceptibility $\chi_{\perp \perp}$ diverges as $\Delta \rightarrow 1^{+}$; at this value of $\Delta$ the excitation gap disappears. The excitation spectrum stays gapless throughout the planar regime $|\Delta| \leq 1$. The direct parallel suscepti- bility $\chi_{z z}$, which was determined rigorously by means of Bethe ansatz calculations, is given by the expression ${ }^{12}$

$$
\chi_{z z}=\mu /[\pi(\pi-\mu) \sin \mu], \cos \mu=-\Delta .
$$

It diverges as $\Delta \rightarrow 1^{-}$, where the ferromagnetic long-range order sets in.

From the results for the $X X Z$ model correlation functions as obtained in the framework of the Luttinger model, ${ }^{34}$ it can be concluded that the perpendicular susceptibility $\chi_{\perp \perp}(q)$ diverges with a power law as $q \rightarrow 0$ for all $|\Delta| \leq 1$, with a $\Delta$-dependent exponent. ${ }^{37}$ The exact nature of the divergence is, however, established only for $\Delta=0$ [our result (3.5b)] and for $\Delta=1$ as explained above. Correspondingly, one can conclude that $\chi_{z z}(q)$ in the regime $-1 \leq \Delta<0$ has a power-law divergence for $q \rightarrow \pi$, also with a $\Delta$-dependent exponent. For $\Delta=0$, the divergence is logarithmic [see (4.2) for the exact result]. The perpendicular staggered susceptibility $\bar{\chi}_{11}$, which is expected to be finite for all $|\Delta| \leq 1$ has not been determined except at $\Delta=1$, where $\bar{\chi}_{\perp \perp}=\frac{1}{4}$ according to (4.12b), and at $\Delta=-1$, where $\bar{\chi}_{11}=1 / \pi^{2}$ according to (4.13) and obvious symmetry properties. Thus, our result $(2.44)$ for $\bar{\chi}_{\perp \perp}$ at $\Delta=0$ is an important nontrivial addition to existing results.

In the regime $\Delta<-1$, finally, the system exhibits antiferromagnetic long-range order. Here $\chi_{z z}=0$ because the spectrum has a gap and $M_{z}$ is a conserved quantity with eigenvalue zero in the ground state. No further exact susceptibility results are known except in the limit $\Delta \rightarrow-\infty$, where $H_{X X Z}$ become equivalent to $H_{A-X Y}$ with $h=0$ and $\gamma=1$.

We now return to $\Delta=0$, for which case we have produced a precise numerical value of the perpendicular staggered susceptibility

$$
\left(\bar{\chi}_{11}\right)_{X Y}=0.07556 \ldots \text {. }
$$

This quantity was already previously the object of theoretical investigations. In a recent study, Duxbury et al. ${ }^{23}$ determined $\left(\bar{\chi}_{11}\right)_{X Y}$ approximately by means of extrapolations based on the numerical calculations of the eigenstates of $H_{X Y}$ for systems with up to $N=10$ spins. They predicted the value $\left(\bar{\chi}_{\perp \perp}\right)_{X Y}=0.055 \pm 0.01$. Very recently, Bonner ${ }^{38}$ applied a different extrapolation scheme on the finite-chain data used for Ref. 23, leading to the following prediction: $\left(\bar{\chi}_{1 \perp}\right)_{X Y} \simeq 0.117$. Both finite-chain results deviate considerably from the value (4.14), in opposite directions. ${ }^{39}$ Such large uncertainties in the finite-chain predictions indicate that the $N \rightarrow \infty$ behavior of the $T=0$ transverse susceptibility of this model is governed by subtleties which easily escape the analysis of finite systems.

One drastic finite-size effect which makes extrapolation procedures for the susceptibility in the low-temperature limit extremely difficult can be described as follows: The $T=0$ magnetization function $M_{\perp}\left(h_{\perp}\right)$ of a finite $X Y$ antiferromagnetic system $(J<0)$ consists of portions of smooth curve interrupted by a finite number of steps which reflect level crossings at the bottom of the spectrum. This situation is illustrated in Fig. 1 of Ref. 20. The transverse susceptibility of a finite system of $N$ spins is given by the expression 


$$
\left(\chi_{\perp \perp}^{(N)}\right)_{X Y, J<0}=2 N \sum_{\lambda(\neq G)} \frac{\left|\left\langle\lambda\left|M_{\perp}\right| G\right\rangle\right|^{2}}{E_{\lambda}-E_{G}},
$$

where $|G\rangle$ is the nondegenerate ground-state wave function with energy $E_{G}$, assuming $N$ is even, and $\lambda$ runs over all $2^{N}-1$ excited states of $H_{X Y}$ with $J<0$. (For odd $N$ where the ground state is twofold degenerate, the calculation proceeds along slightly different lines.) The susceptibility value obtained from (4.15) corresponds to the initial slope of the first smooth portion of the finite-system magnetization curve $M_{\perp}\left(h_{\perp}\right)$. In the thermodynamic limit $N \rightarrow \infty$, the number of steps in the function $M_{1}\left(h_{1}\right)$ increases like $N / 2$, whereas the step size tends to zero such that the result is a smooth curve with a single nonanalyticity at the critical field $h_{1}^{(c)}$. The important point in the present context is that the location of the first step approaches $h=0$ as $N \rightarrow \infty$. Hence, it is not clear that (4.15) should extrapolate to the susceptibility

$$
\left(\chi_{\perp \perp}\right)_{X Y, J<0}=\left(\bar{\chi}_{\perp \perp}\right)_{X Y, J>0}
$$

of the infinite system. It is likely that (4.15) converges to a lower value. This is, in fact, known to be the case for the susceptibility $\left(\chi_{z z}\right)_{X Y}$ where the smooth portions in the function $M_{z}\left(h_{z}\right)$ for finite $N$ are strictly horizontal. Here, Eq. (4.15) would predict $\left(\chi_{z z}\right)_{X Y}=0$ for all $N$, whereas the exact result for $N=\infty$ is $\left(\chi_{z z}\right)_{X Y}=1 / \pi$ according to (4.2).

In a different context, it was found ${ }^{15}$ that under such circumstances a more accurate value for the susceptibility could be obtained by extrapolating the slope of the line connecting the origin with the midpoint of the first step in the function $M(h)$. At present, however, there are not sufficient data available to implement this scheme for $\left(\bar{\chi}_{\perp \perp}\right)_{X Y}$.

\section{ACKNOWLEDGMENTS}

We thank J. C. Bonner, B. M. McCoy, J. H. H. Perk, and $\mathrm{H}$. Thomas for useful discussions. This research was supported in part by the National Science Foundation under Grant No. PHY-81-9110-A-01.

\section{APPENDIX}

In this appendix we list the $c_{j, k}^{(n)}$ coefficients for $n=1,2$ and $j \leq 20$. The very great accuracy of such a high order of expansion is important in obtaining a requisite degree of accuracy in the solution to the ODE (2.16) because of the (regular) singular point at $z=0$. We have actually calculated the $c_{j, k}^{(n)}$ for $n$ up to 10 but shall not list the results here because of their length. Because the denominators have a more systematic factorization property than the numerators, we shall list the $c_{j, k}^{(n)}$ in a form in which the denominators, but not the numerators, are factored. For $n=1$ :

$$
\begin{aligned}
& c_{2,0}^{(1)}=-\frac{1}{3^{1}}, \\
& c_{3,1}^{(1)}=\frac{1}{2^{1} 3^{1}}, \\
& c_{4,0}^{(1)}=-\frac{1}{3^{2} 5^{1}},
\end{aligned}
$$

$c_{5,1}^{(1)}=\frac{7}{2^{1} 3^{3} 5^{1}}$

$c_{6,0}^{(1)}=-\frac{2}{3^{3} 5^{1} 7^{1}}$,

$c_{6,2}^{(1)}=-\frac{1}{2^{2} 3^{3}}$,

$c_{7,1}^{(1)}=\frac{41}{2^{2} 3^{4} 5^{1} 7^{1}}$,

$c_{8,0}^{(1)}=-\frac{1}{3^{3} 5^{2} 7^{1}}$,

$c_{8,2}^{(1)}=-\frac{1}{2^{1} 3^{2} 5^{2}}$,

$c_{9,1}^{(1)}=\frac{19}{2^{2} 3^{4} 5^{3}}$,

$c_{9,3}^{(1)}=\frac{1}{2^{3} 3^{5}}$,

$c_{10,0}^{(1)}=-\frac{2}{3^{5} 5^{1} 7^{1} 11^{1}}$,

$c_{10,2}^{(1)}=-\frac{3671}{2^{1} 3^{6} 5^{3} 7^{2}}$,

$c_{11,1}^{(1)}=\frac{181}{2^{2} 3^{4} 5^{3} 7^{1} 11^{1}}$,

$c_{11,3}^{(1)}=\frac{11}{2^{5} 3^{4} 5^{2}}$,

$c_{12,0}^{(1)}=-\frac{1382}{3^{6} 5^{3} 7^{2} 11^{1} 13^{1}}$,

$c_{12,2}^{(1)}=-\frac{251}{2^{1} 3^{7} 5^{3} 7^{1}}$,

$c_{12,4}^{(1)}=-\frac{1}{2^{4} 3^{7}}$,

$c_{13,1}^{(1)}=\frac{931.687}{2^{1} 3^{7} 5^{4} 7^{3} 11^{1} 13^{1}}$,

$c_{13,3}^{(1)}=\frac{9217}{2^{5} 3^{5} 5^{4} 7^{2}}$,

$c_{14,0}^{(1)}=-\frac{4}{3^{6} 5^{2} 7^{1} 11^{1} 13^{1}}$,

$c_{14,2}^{(1)}=-\frac{15685151}{2^{4} 3^{9} 5^{3} 7^{3} 11^{2}}$,

$c_{14,4}^{(1)}=-\frac{7}{2^{5} 3^{6} 5^{2}}$,

$c_{15,1}^{(1)}=\frac{87133}{2^{3} 3^{7} 5^{3} 7^{3} 11^{1} 13^{1}}$,

$c_{15,3}^{(1)}=\frac{235439}{2^{5} 3^{8} 5^{5} 7^{2}}$,

$c_{15,5}^{(1)}=\frac{1}{2^{5} 3^{9}}$,

$c_{16,0}^{(1)}=-\frac{3617}{3^{7} 5^{4} 7^{2} 11^{1} 13^{1} 17^{1}}$,

$c_{16,2}^{(1)}=-\frac{1120501}{2^{2} 3^{10} 5^{2} 7^{1} 11^{2} 13^{2}}$, 


$$
\begin{aligned}
& c_{16,4}^{(1)}=-\frac{1399}{2^{5} 3^{7} 5^{3} 7^{2}}, \\
& c_{17,1}^{(1)}=\frac{114811903}{2^{3} 3^{10} 5^{5} 7^{3} 11^{1} 13^{1} 17^{1}}, \\
& c_{17,3}^{(1)}=\frac{2122430231}{2^{5} 3^{10} 5^{5} 7^{4} 11^{2}}, \\
& c_{17,5}^{(1)}=\frac{17}{2^{7} 3^{8} 5^{2}}, \\
& c_{18,0}^{(1)}=-\frac{87734}{3^{9} 5^{3} 7^{3} 11^{1} 13^{1} 17^{1} 19^{1}}, \\
& c_{18,2}^{(1)}=-\frac{18171487927}{2^{4} 3^{10} 5^{6} 7^{3} 11^{2} 13^{2}}, \\
& c_{18,4}^{(1)}=-\frac{1}{2^{6} 3^{11}}, \\
& c_{19,1}^{(1)}=\frac{5187560281}{2^{3} 3^{11} 5^{5} 7^{4} 11^{1} 13^{1} 17^{1} 19^{1}}, \\
& c_{19,3}^{(1)}=\frac{288443993599}{2^{7} 3^{12} 5^{5} 7^{3} 11^{2} 13^{2}}, \\
& c_{19,5}^{(1)}=\frac{79021}{2^{9} 3^{9} 5^{4} 7^{2}}, \\
& c_{20,0}^{(1)}=-\frac{349222}{3^{9} 5^{5} 7^{2} 11^{2} 13^{1} 17^{1} 19^{1}}, \\
& c_{20,2}^{(1)}=-\frac{75756058808}{3^{12} 5^{5} 7^{3} 11^{2} 13^{2} 17^{2}}, \\
& c_{20,4}^{(1)}=-\frac{6601254637}{2^{2} 3^{12} 5^{7} 7^{4} 11^{2}}, \\
& c_{20,6}^{(1)}=-\frac{1}{2^{6} 3^{10} 5^{1}} . \\
&
\end{aligned}
$$

For $n=2$ :

$$
\begin{aligned}
& c_{2,0}^{(2)}=-\frac{4}{3^{1} 5^{1}}, \\
& c_{4,0}^{(2)}=\frac{4}{3^{2} 5^{2} 7^{1}}, \\
& c_{5,1}^{(2)}=-\frac{1}{2^{1} 3^{3} 5^{1}}, \\
& c_{6,0}^{(2)}=\frac{8}{3^{3} 5^{3} 7^{1}}, \\
& c_{7,1}^{(2)}=-\frac{16}{3^{4} 5^{3} 7^{1}}, \\
& c_{8,0}^{(2)}=\frac{292}{3^{4} 5^{4} 7^{2} 11^{1}}, \\
& c_{9,1}^{(2)}=-\frac{1}{2^{1} 3^{4} 5^{4} 7^{1}},
\end{aligned}
$$

$$
\begin{aligned}
& c_{10,0}^{(2)}=-\frac{8}{3^{1} 5^{5} 7^{2} 11^{1} 13^{1}}, \\
& c_{10,2}^{(2)}=-\frac{1}{2^{2} 3^{6} 5^{3}} \text {, } \\
& c_{11,1}^{(2)}=\frac{5062}{3^{6} 5^{5} 7^{3} 11^{1}} \text {, } \\
& c_{12,0}^{(2)}=-\frac{14632}{3^{6} 5^{6} 7^{3} 11^{1} 13^{1}}, \\
& c_{12,2}^{(2)}=-\frac{1}{2^{2} 3^{6} 5^{2} 7^{2}}, \\
& c_{13,1}^{(2)}=\frac{28031}{2^{2} 3^{5} 5^{6} 7^{3} 11^{1} 13^{1}}, \\
& c_{14,0}^{(2)}=-\frac{21104}{3^{7} 5^{7} 7^{2} 11^{1} 13^{1} 17^{1}}, \\
& c_{14,2}^{(2)}=-\frac{893}{3^{9} 5^{6} 7^{3}} \text {, } \\
& c_{15,1}^{(2)}=\frac{20054}{3^{8} 5^{6} 7^{4} 11^{1} 13^{1}}, \\
& c_{15,3}^{(2)}=-\frac{1}{2^{3} 3^{9} 5^{5}} \text {, } \\
& c_{16,0}^{(2)}=-\frac{461068}{3^{8} 5^{8} 7^{4} 11^{2} 13^{1} 17^{1} 19^{1}}, \\
& c_{16,2}^{(2)}=\frac{215189}{2^{1} 3^{10} 5^{7} 7^{3} 11^{2}}, \\
& c_{17,1}^{(2)}=-\frac{33988987}{2^{2} 3^{10} 5^{8} 7^{4} 11^{1} 13^{1} 17^{1}}, \\
& c_{17,3}^{(2)}=-\frac{17}{2^{5} 3^{10} 5^{4} 7^{2}}, \\
& c_{18,0}^{(2)}=\frac{93122584}{3^{9} 5^{9} 7^{4} 11^{2} 13^{1} 17^{1} 19^{1}}, \\
& c_{18,2}^{(2)}=\frac{3180719}{2^{1} 3^{10} 5^{8} 7^{2} 11^{2} 13^{2}}, \\
& c_{19,1}^{(2)}=-\frac{156785343061}{3^{11} 5^{9} 7^{5} 11^{3} 13^{1} 17^{1} 19^{1}}, \\
& c_{19,3}^{(2)}=-\frac{247}{2^{2} 3^{12} 5^{5} 7^{4}}, \\
& c_{20,0}^{(2)}=\frac{142878941144}{3^{10} 5^{10} 7^{5} 11^{2} 13^{2} 17^{1} 19^{1} 23^{1}} \text {, } \\
& c_{20,2}^{(2)}=\frac{2468009}{2^{1} 3^{11} 5^{8} 7^{3} 11^{2} 13^{2}}, \\
& c_{20,4}^{(2)}=-\frac{1}{2^{4} 3^{12} 5^{7}} \text {. }
\end{aligned}
$$

*Present address: Department of Physics, University of Rhode Island, Kingston, RI 02881.

${ }^{1}$ Experimental studies on quasi-1D magnetic compounds have been reviewed, e.g., in M. Steiner, J. Villain, and C. G. Windsor, Adv. Phys. 25, 87 (1976); L. J. De Jongh and A. R. Miedema, ibid. 23, 1 (1974); L. J. De Jongh, J. Appl. Phys. 49, 1305 (1978).
2E. Lieb, T. Schultz, and D. Mattis, Ann. Phys. (N.Y.) 16, 407 (1961).

${ }^{3}$ S. Katsura, Phys. Rev. 127, 1508 (1962); 129, 2835 (1963).

${ }^{4}$ M. E. Fisher, J. Math. Phys. 4, 124 (1963).

${ }^{5}$ T. Niemeijer, Physica (Utrecht) 36, 377 (1967).

6B. M. McCoy, Phys. Rev. 173, 531 (1968).

${ }^{7}$ E. Barouch, M. Dresden, and B. M. McCoy, Phys. Rev. A 2, 
1075 (1970); E. Barouch and B. M. McCoy, ibid. A 3, 786 (1971); 3, 2137 (1971); B. M. McCoy, E. Barouch, and D. B. Abraham, ibid. A 4, 2331 (1971).

${ }^{8}$ S. Katsura, T. Horiguchi, and M. Suzuki, Physica (Utrecht) 46, 67 (1970).

${ }^{9}$ M. Suzuki, Prog. Theor. Phys. 46, 1337 (1971).

10P. Pfeuty, Ann. Phys. (N.Y.) 57, 79 (1970).

11J. Lajzerowicz and P. Pfeuty, Phys. Rev. B 11, 4560 (1975).

${ }^{12}$ C. N. Yang and C. P. Yang, Phys. Rev. 147, 303 (1966); 150, 321 (1966); 150, 327 (1966); 151, 258 (1966).

13J. des Cloizeaux and M. Gaudin, J. Math. Phys. 7, 1384 (1966).

${ }^{14}$ V. M. Kontorovich and V. M. Tsukernik, Zh. Eksp. Teor. Fiz. 53, 1167 (1967) [Sov. Phys.-JETP 26, 687 (1968)]; J. H. H. Perk, H. W. Capel, M. J. Zuilhof, and T. J. Siskens, Physica 81A, 319 (1975).

15J. C. Bonner and M. E. Fisher, Phys. Rev. 135, A640 (1964).

${ }^{16}$ R. B. Griffiths, Phys. Rev. 133, A768 (1964).

${ }^{17}$ H. W. Capel and J. H. H. Perk, Physica 87A, 211 (1977); J. H. H. Perk and H. W. Capel, ibid. 89A, 265 (1977); 100A, 1 (1980).

${ }^{18}$ T. N. Tommet and D. L. Huber, Phys. Rev. B 11, 450 (1975).

${ }^{19}$ E. J. van Dongen and H. W. Capel, Physica 84A, 285 (1976).

20J. Kurmann, G. Müller, H. Thomas, M. W. Puga, and H. Beck, J. Appl. Phys. 52, 1968 (1981).

21J. Kurman, H. Thomas, and G. Müller, Physica 112A, 235 (1982); J. H. Taylor and G. Müller, Phys. Rev. B 28, 1529 (1983).

${ }^{22}$ G. Müller, H. Thomas, M. W. Puga, and H. Beck, J. Phys. C 14, 3399 (1981).

23P. M. Duxbury, J. Oitmaa, M. N. Barber, A. van Der Bilt, K. O. Joung, and R. L. Carlin, Phys. Rev. B 24, 5149 (1981).

${ }^{24}$ B. M. McCoy, J. H. H. Perk, and R. E. Shrock, Nucl. Phys. B220, [FS8], 35 (1983).

${ }^{25}$ B. M. McCoy, J. H. H. Perk, and R. E. Shrock, Nucl. Phys. B220, [FS8], 269 (1983).

${ }^{26}$ G. Müller and R. E. Shrock, Phys. Rev. Lett. 51, 219 (1983); Phys. Rev. B 29, 288 (1984).

${ }^{27}$ G. Müller and R. E. Shrock, J. Appl. Phys. 55, 1874 (1984). This paper extends the work of Ref. 26 to the case $n \neq 0$.

${ }^{28}$ H. G. Vaidya and C. A. Tracy, Physica 92A, 1 (1978).

${ }^{29}$ The only exception is the following: If the operator $M_{\xi}(q)$ of (1.1b) commutes with the Hamiltonian for some value of $q$, then the classical fluctuation-dissipation theory still holds for $\chi_{\xi \xi}(q)$ at $T>0$.

${ }^{30}$ In Fourier space, this symmetry property is manifested as the detailed-balance condition $S_{\xi \xi}(q,-\omega)=\exp (-\beta \omega) S_{\xi \xi}(q, \omega)$ for the dynamic structure factor.

${ }^{31}$ This derivation requires that the functions $\Xi_{n}(t)$ be analytic in the lower-half complex $t$ plane including the real axis. For the TI and $X Y$ models this condition is satisfied since the $\Xi_{n}(t)$ are known to be entire. See J. Ginibre, Commun. Math. Phys. 10, 140 (1968); W. Greenburg, ibid. 11, 3147 (1969); H. Araki, ibid. 14, 120 (1969); D. Ruelle, Statistical Mechanics (Benjamin, New York, 1969). See also Ref. 17.

${ }^{32}$ For $q=m \pi / M$ and even $m, \chi_{x x}(q)$ can also be expressed as a convergent series. In this case, however, each coefficient is obtained as the time integral over the sum of $M$ correlation functions $X_{n}(-i \tau)$.

${ }^{33}$ It may be noted that a related result was obtained in M. E. Fisher and R. J. Burford, Phys. Rev. 156, 583 (1967) for the $q$-dependent susceptibility of the $2 \mathrm{D}$ Ising model on a square lattice. By the Suzuki mapping (Ref. 9) the 1D transversing Ising model at critical field may be related to (an infinitely anisotropic) $2 \mathrm{D}$ Ising model at the critical temperature. Since the leading term in the divergent susceptibility is independent of the anisotropy, at least for finite anisotropy, it is plausible that these two results should agree.

${ }^{34}$ A. Luther and I. Peschel, Phys. Rev. B 12, 3908 (1975); see also H. C. Fogedby, J. Phys. C 11, 4767 (1978).

${ }^{35}$ G. Müller, H. Thomas, H. Beck, and J. C. Bonner, Phys. Rev. B 24, 1429 (1981).

${ }^{36}$ Y. Tanaka and N. Uryû, Phys. Rev. B 21, 1994 (1980).

${ }^{37}$ See Ref. 22 for a discussion of the divergent susceptibilities of the $X X Z$ model.

38J. C. Bonner, lecture notes, North Atlantic Treaty Organization (NATO) summer school, Italy, 1983 (to be published).

${ }^{39}$ For completeness we should also mention that the approach reported in Ref. 22, which predicted the divergent part of $\chi_{11}(q)_{X Y}$ to very high accuracy, as discussed in Sec. III, yields an even higher value for the staggered susceptibility: $\left(\bar{\chi}_{11}\right)_{X Y}=0.188 \ldots$ This large deviation from (4.14) can be understood to reflect the importance of high-energy excitations for this quantity, which are systematically neglected in the approach of Ref. 22. 